\title{
Anwendung der Disk-Elektrophorese zur Trennung der Isoenzyme der Alkalischen Phosphatase')
}

\author{
Von A. Akhtar, A. Hansen und K. H. Kärcher \\ Aus der Strablenklinik der Universität Heidelberg (Direktor: Prof. Dr. Dr. b. c. J. Becker)
}

(Eingegangen am 27. Oktober 1967)

\begin{abstract}
Mindestens drei Haupttypen Alkalischer Phosphatase-Isoenzyme von Ratten können durch Disk-Elektrophorese getrennt und Leber, Niere und Knochen zugeordnet werden. Die Bestrahlung dieser Organe bewirkt keine Veränderung des AP-Isoenzym-Musters im Serum.

Disk-elektrophoretische Untersuchungen mit Polyacrylamidgel an normalen Seren von Menschen zeigen eine unterschiedliche Verteilung dieser Isoenzyme und fehlende Unterschiede bei verschiedener Organmetastasierung. Deshalb kann diese Trennung nicht zur Differentialdiagnose von Leber- und Knochenmetastasen verwendet werden.

At least three main types of alkaline phosphatase isoenzymes from rats were separated by disc electrophoresis. They were identified as the liver, kidney and bone types. The irradiation of these organs did not produce any change in the pattern of alkaline phosphatase isoenzymes of the serum.

Disc electrophoretic investigations of normal human sera with the aid of polyacrylamide gel does show no constancy in the distribution pattern of these isoenzymes and no differences in patients with metastasis of various organs. The separation is therefore of no use for the differential diagnosis of liver and bone metastases.
\end{abstract}

Die Entwicklung der Disk-Elektrophorese an Acrylamidgel $(1,2)$ und ihre Anwendung auf die Trennung von Serum-Proteinen $(3,4)$ erlauben es, noch bessere Auftrennungsergebnisse zu erzielen, als dies mit den anderen elektrophoretischen Methoden $(5,6)$ möglich ist. TASIVELL und JefFers (7) trennten AlkalischePhosphatase-Isoenzyme (AP-Isoenzyme) $(1,2)$ aus Seren von gesunden Personen und Patienten mit Leber-, Gallen- und Knochenkrankheiten elektrophoretisch an Stärkegel. Sie kamen zu dem Ergebnis, $\mathrm{da} \beta$ aus solchen Untersuchungen diagnostische Aussagen abgeleitet werden können, da bei Kranken die Konzentration einzelner AP-Isoenzyme anders sei als bei Gesunden. Die Notwendigkeit einer Differentialdiagnose (8) bei Patienten mit verschiedenen Metastasen, vor allem Leber- und Knochenmetastasen, gab den Ansto $B$, durch AP-Isoenzym-Trennung mit der DiskElektrophorese Untersuchungen in ähnlicher Weise mit Polyacrylamidgel zu beginnen.

Das Problem liegt in der Lokalisierung der organspezifischen AP-Isoenzyme mit dieser Methode. Untersuchungen mit ähnlichen Zielen wie die von TASWELL und JEFFERs (7.) sollten $\mathrm{zu}$ besseren Ergebnissen führen, wenn es gelingt, die Trennungen quantitativ auszuwerten $(9,10)$.

Wir untersuchten die AP-Isoenzyme in Seren bestrahlter Ratten und benutzten dieses Modell, da man eine Ausschwemmung der organspezifischen AP-Isoenzyme aus den Zellen des bestrahlten Organs in das Serum als Folge des Strahleninsultes annehmen kann.

\section{Material und Methode}

Zur Trennung verwendeten wir Glasröhrchen von $7 \mathrm{~cm}$ Länge mit einem inneren Durchmesser von $5 \mathrm{~mm}$ im Acrylamid-Säulenelektrophoresegerät der Firma Shandon Labortechnik GmbH. Die

1) Der Trivialname Alkalische Phosphatase wird hier gebraucht für das Enzym Orthophosphorsäuremonoester Phosphohydrolase (EC 3.1.3.1).
Polymerisationsmethode des Trenngels lehnt sich im großen ganzen an die von Davis (4) an. Das Trenngel wird aus einer 25 proz. statt 28 proz. Acrylamidlösung hergestellt und mit Peroxyd-TEMED-Katalysator polymerisiert ${ }^{2}$ ). Die Polymerisation des Sammelgels erzielten wir photokatalytisch mit Riboflavin und Phosphatpuffer (12):

1 Teil der Lösung von $25,6 \mathrm{~m} l 1 \mathrm{M} \mathrm{H}_{3} \mathrm{PO}_{4}$ und $5,7 \mathrm{~g}$ Trịs ad $100 \mathrm{~m} l$ bidest. Wasser

2 Teile der Lösung von 10,0 g Acrylamid und 2,5 g N,N'-Metyhlenbisacrylamid ad $100 \mathrm{~m} l$ bidest. Wasser

1 Teil der Lösung von 4,0 mg Riboflavin ad $100 \mathrm{~m} /$ bidest. Wasser und

4 Teile der 40 proz. Saccharoselösung.

Mit diesem. Gemisch wurde der Raum über dem Trenngel ausgespült. Die Spüllösung wurde verworfen, $1 \mathrm{~cm}$ des Gemisches mit Wasser überschichtet und $20 \mathrm{Min}$. dicht an einer Quecksilberlampe photopolymerisiert. Die Polymerisation war beendet, nachdem das Sammelgel durch und durch milchig-trüb geworden war. Davis (4) benutzte den Riboflavin-TEMED-Katalysator für diese Polymerisation.

Die Pufferlösung für die Trennung wurde hergestellt aus $14,4 \mathrm{~g}$ Glycin und 3,0 g Tris in $1000 \mathrm{~m} /$ dest. Wasser. Die übrigen Lösungen entsprachen dèn von DAvis (4) angegebenen.

Für jede Untersuchung am Menschen verwenden wir $0,01-0,02 \mathrm{ml}$ frisches unverdünntes Serum. Von gesundèn Vergleichspersonen und Tumorpatienten wird venöses Blut entnommen, zentrifugiert und sofort untersucht oder bei $-20^{\circ}$ gehalten und innerbalb einer Woche analysiert. Nach Literaturangaben $(7,13,14)$ beträgt die Stabilität bei $-20^{\circ}$ mehrere Monate. Die elektophoretische Trennung dauert gewöhnlich $90-95 \mathrm{Min}$. (2 mA/Röhrchen).

Extrakte verschiedener Organe von Albinoratten werden in physiol. NaCl-Lösung homogenisiert, 50 Min. zentrifugiert (12 000 U./Min.) und die überstehende Flüssigkeit dekantiert. Für $0,1 \mathrm{~g}$ Niere verwenden wir $2 \mathrm{~m} l$ physiol. $\mathrm{NaCl}$-Lösung, für $0,1 \mathrm{~g}$ Knochen $2 \mathrm{~m} l$ und für $0,2 \mathrm{~g}$ Leber $1 \mathrm{~m} l$, die Ratten-Seren werden unverdünnt verwendet.

Die aufgetragene Menge des Extraktes variiert zwischen $0,005 \mathrm{ml}$ und $0,02 \mathrm{~m} /$ für jede Trennung. Bei einer Mischung verschiedener Extrakte wird darauf geachtet, daß etwa $0,005 \mathrm{~m} l$ jeder ExtraktKomponente für jede Trennung vorlag.

Zur Anfärbung wird die Gelsäule in eine frisch bereitete unfiltrierte Lösung von $50 \mathrm{mg}$ Natrium-naphthylphosphat und $100 \mathrm{mg}$

2) Abkürzungen: $\mathrm{AP}=$ Alkalische Phosphatase; Peroxyd = Ammoniumpersulfat, TEMED $\doteq \mathrm{N}, \mathbf{N}_{2} \mathrm{~N}^{\prime}, \mathrm{N}^{\prime}$-Tetramethyläthylendiamin; $\mathrm{OD}=$ Oberflächendosis; $\mathrm{QHD}=$ Quelle-Hautdistanz. 
Variaminblausalz B konz. der Firma Merck in $100 \mathrm{~m} / 2$ proz. Veronal-Natriumlösung gestellt (15). Das Gel bleibt etwa 30 Min. bei $37^{\circ}$ in dieser Lösung. Danach wird es aus dem Brutschrank genommen, mit Wasser gewaschen und in klcinen wassergefüllten Reagenzgläsern aufbewahrt. Das Gel kann im Wasser unbeschränkte Zeit aufgehoben werden.

Als Strahlung verwendeten wir die Quelle eines Caesa-Gammatrons $(0,662 \mathrm{MeV}, 2000 \mathrm{C})$. Es wurde mit eingeblendeten Feldern bei verschiedenen Tieren entweder die Leber, Niere oder der Knochen des Beckenbereiches bestrahlt. Verständlicherweise ließ es sich hierbei nicht vermeiden, Umgebungsgewebe mitzubestrahlen. Die Bestrahlungsbedingungen für die jeweilige Lokalisation wurde folgendermaßen gewählt: $1500 \mathrm{R}$ OD Einzeitbestrahlung, Feldgr.

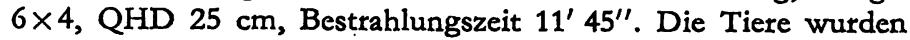
3 bzw. 18 Stdn. nach der Bestrahlung durch Entbluten mittels Punktion der Aorta abdominalis gëtötet. Hierzu erhielten sie $16 \mathrm{mg}$ Trapanal $^{3}$ ) in $0,3 \mathrm{~m} / \mathrm{i}$. p. Bei der Punktion der Aorta kann völlig hämolysefreies arterielles Blut entnommen werden.

\section{Ergebnisse und Diskussion}

\section{Lokalisierung von organspezifischen $A P$-Isoenz ymen}

Die Extrakte wurden 160-180 Min. bei $4^{\circ}$ und einer Stromstärke von $2 \mathrm{~mA} /$ Röhrchen der elektrophoretischen Trennung unterzogen. Jedes der drei Organe ergab ein unterschiedliches AP-Isoenzym-Muster, das man an ihrer Wanderungsgeschwindigkeit und der Bandbreite erkennen kann. Am einfachsten läßt sich das Leber-AP-Isoenzym identifizieren, das am schnellsten wandert. Knochen- und Nieren-AP-Isoenzyme zeigen eine verbreiterte Zone, wenn sie als Gemisch laufen. Nur in großer Verdünnung beobachtet man zwei Banden (Abb. 1). Auf diese Weise kann man die ver-

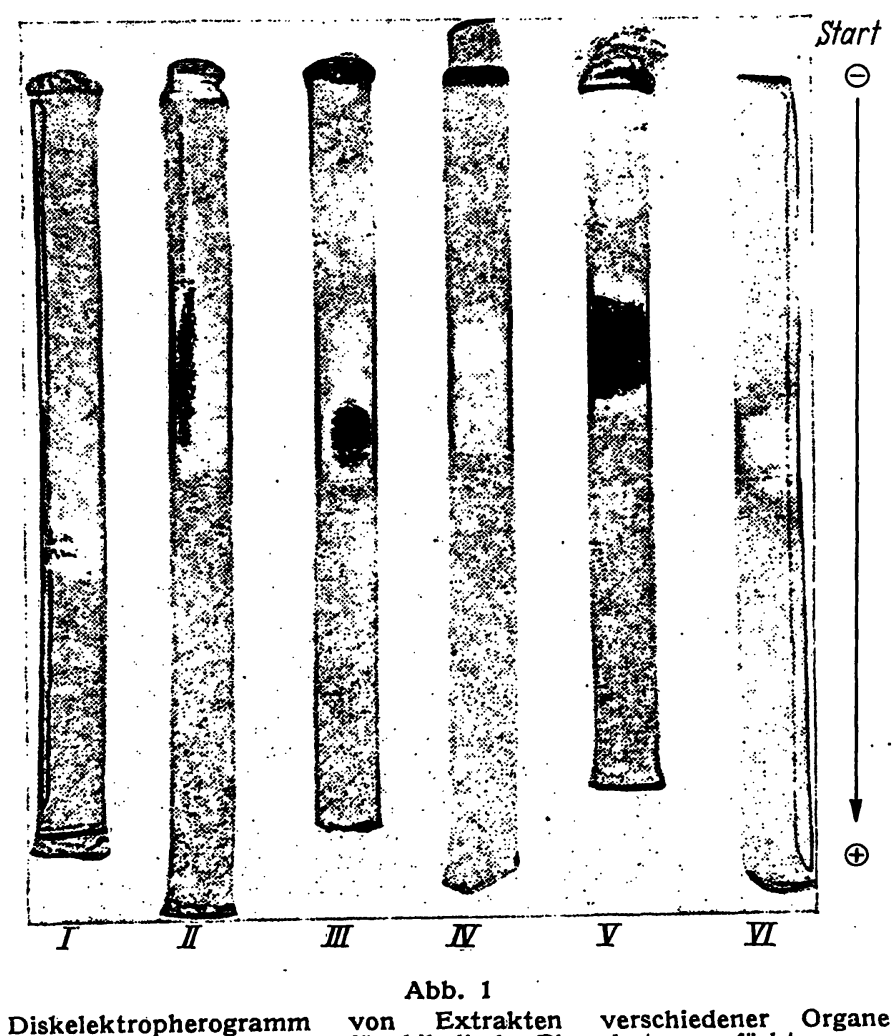
bestrahlter Ratten, für Alkalische Phosphate angefärbt.

I = Leberextrakt, II = Gemisch von Leber und Knochen, III = Knochenextrakt, IV = Gemisch von Leber und Niere, $V=$ Nierenextrakt, VI $=$ Rattenserum

3) 5-Äthyl-5-(1' methyl-butyl)-2-thiobarbitursäure. schiedenen AP-Isoenzyme des Serums den entsprechenden Organen zuordnen $(9,10)$. Im Serum ist LeberAP-Isoenzym konzentrierter als im Leberextrakt selbst. Darüber hinaus beobachtet man ein drittes schmales Band zwischen Leber- und Knochenbanden, gelegentlich noch ein oder zwei schmale Banden 1 oder $2 \mathrm{~mm}$ von der Auftragstelle, die in Abbildung 1 nicht $\mathrm{zu}$ erkennen sind, da sie bis zur Fertigstellung der Aufnahme bereits verblaßt sind.

\section{Bestrablungswirkung auf die Serum-AP-Isoenz yme}

Das Serum junger Ratten (max. Gewicht $100 \mathrm{~g}$ ) wird nach 3 Stdn. und 18 Stdn. abgenommen und der DiskElektrophorese parallel zu einer Probe normalen Serums unterworfen. Das AP-Isoenzym-Muster des Serums bestrahlter Ratten wird gegenüber normalem Serum nicht verändert.

\section{$A P$-Isoenz ym-Muster im Serum von Menschen}

Je $0,01 \mathrm{ml}$ Serum von 4 gesunden Personen $(2$ männlich und 2 weiblich) und von einer Patientin mit Lebermetastasen, einer Patientin mit Knochenmetastasen und zwei Patientinnen mit Fernmetastasen maligner Tumoren wird 90-95 Min. bei $4^{\circ}$ der Disk-Elektrophorese unterzogen (Stromstärke: $2 \mathrm{~mA} /$ Röhrchen). Die Gelsäulen werden mit diffusem Licht durchleuchtet und photographiert. Die Negative werden in ein Diapositiv handlicher Größe vergrößert, so daß deren Intensität mit einer Photozelle gemessen werden kann. Zur Auswertung verwendeten wir den Extinktionsschreiber Integraph II der Firma C. Zeiss, Oberkochen/Württ., Lichtfilter FE 60 entsprechend einer Wellenlänge von $600 \mathrm{~nm}$.

Hierdurch gewinnt man aus technischen Gründen zwar kein absolutes $\mathrm{Ma} B$ der AP-Isoenzymkonzentration, aber wohl das relative Verhältnis im betrachteten Serum. Auf diese Weise ist man in der Lage, AP-Isoenzymkonzentrationsverschiebungen von Patienten gegenüber solchen von normalen Personen festzustellen. Wie die Abbildungen und Kurven (Abb. 2-9) zeigen, läßt sich kein Unterschied zwischen normalen Personen und Patienten mit Metastasen feststellen. Sogar die Muster der verschiedenen gesunden Kontrollpersonen sind unterschiedlich, so $\mathrm{da}$ B man keine diagnostische Aussage ableiten kann.

Mindestens sechs verschiedene AP-Isoenzyme werden beobachtet. Nicht alle sind auf den Abbildungen zu erkennen, da die Banden vor den Aufnahmen verblassen. Ihre Zonen werden nicht den Proteinbanden zugeordnet (9). $\mathrm{Da}$ diese Untersuchungen an normalen Seren von Menschen eine unterschiedliche Verteilung der verschiedenen AP-Isoenzyme aufzeigt und bei verschiedenen Metastasierungstypen Unterschiede vermißt werden, verzichteten wir auf eine weitere Bearbeitung dieses Problems.

Die Untersuchungen wurden mit Unterstützung des Bundesministers für wissenschaftliche Forschung, Bad Godesberg, durchgeführt. 


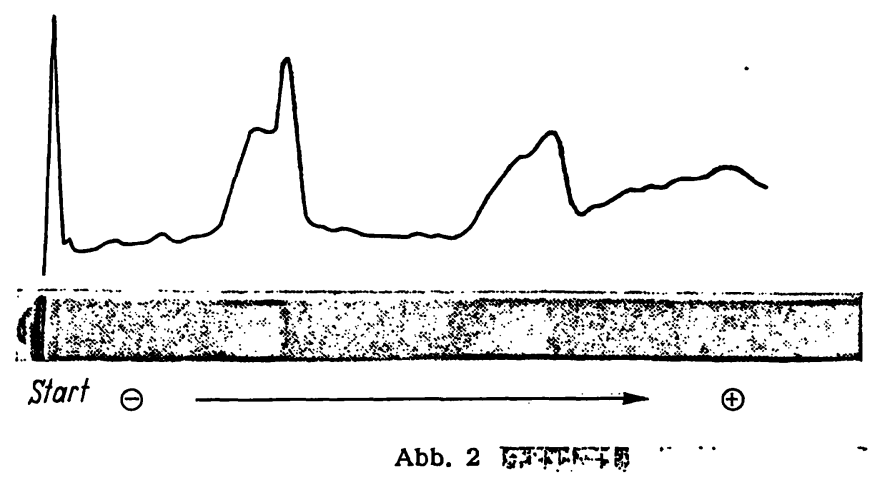

Diskelektropherogramm und Deusitogramm eines Normal-Serums vom Menschen $\left(\sigma^{\circ}, 1\right)$

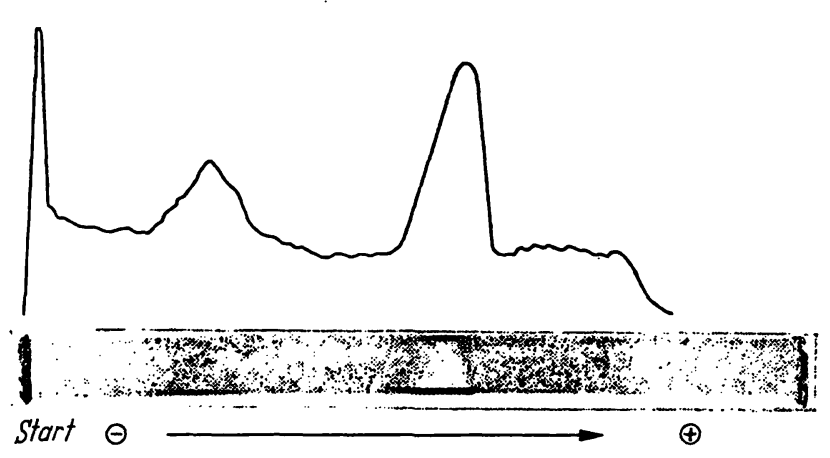

Abb. 3

Diskelektropherogramm und Densitogramm eines Normal-Serums vom Menschen $\left(\sigma^{\prime}, 2\right)$

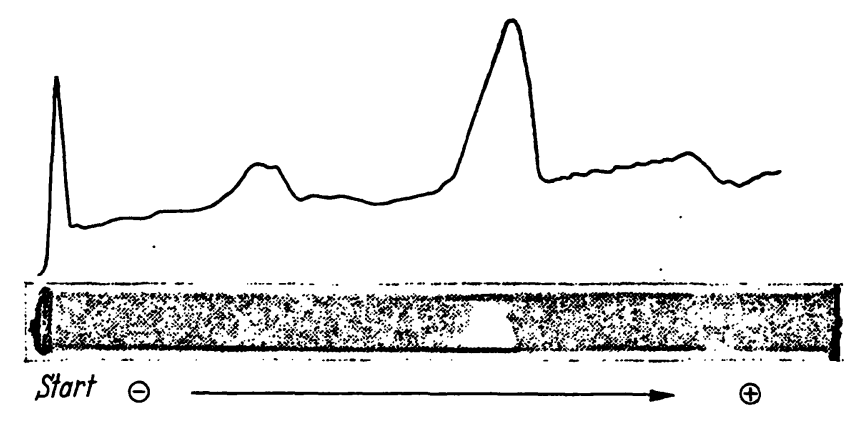

Abb. 4

Diskelektropherogramm und Densitogramm eines Normal-Serums vom Menschen $(\%, 1)$

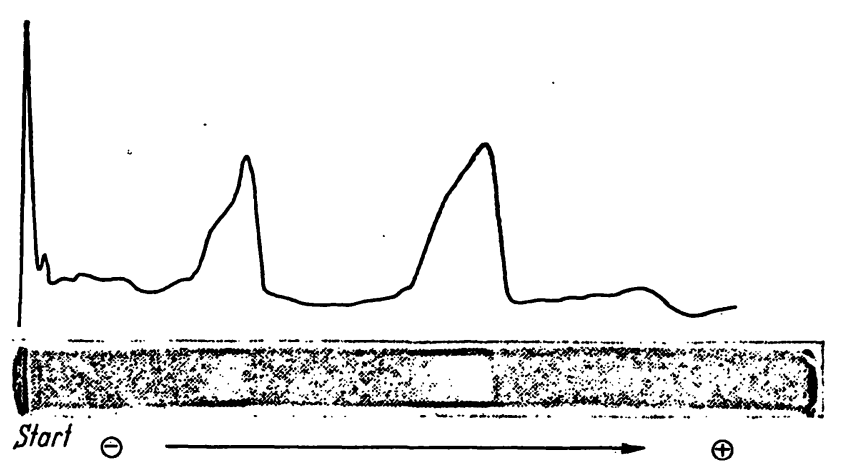

Abb. 5

Diskelektropherogramm und Densitogramm eines Normal-Serums vom Menschen $(0,2)$

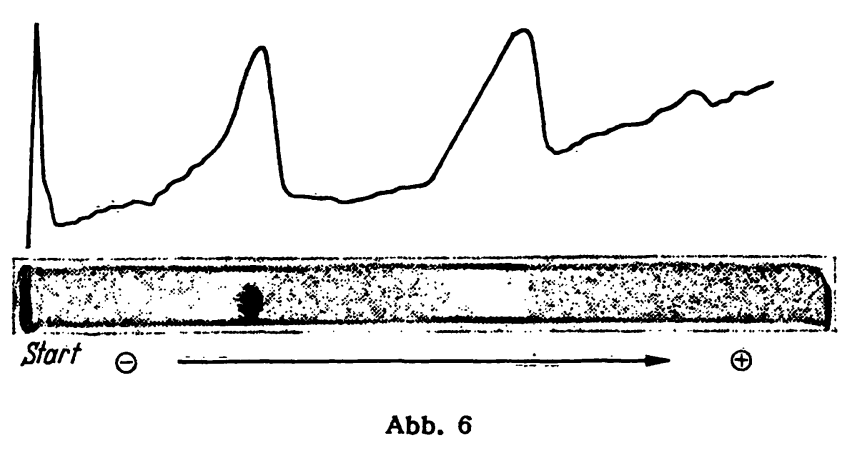

Diskelektropherogramm und Densitogramm des Serums einer Tumorpatientin mit Knochenmetastasen

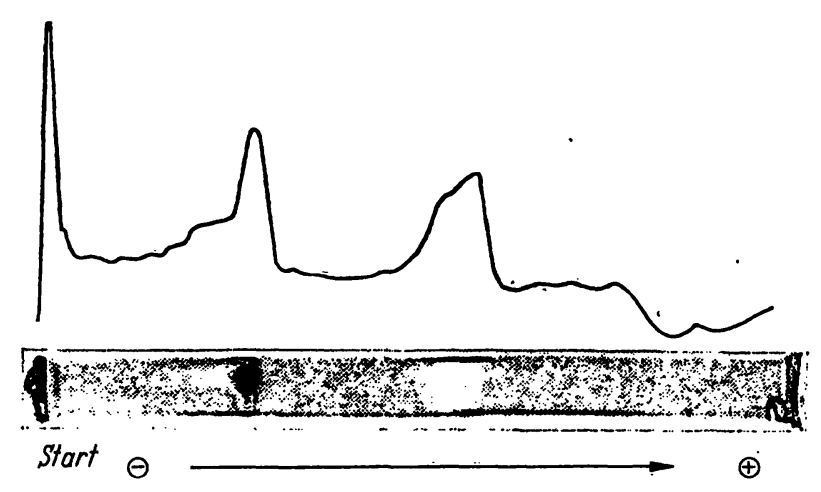

Aḅb. 7

Diskelektropherogramm und Densitogramm des Serums einer Tumorpátientin mit Lebermetastasen

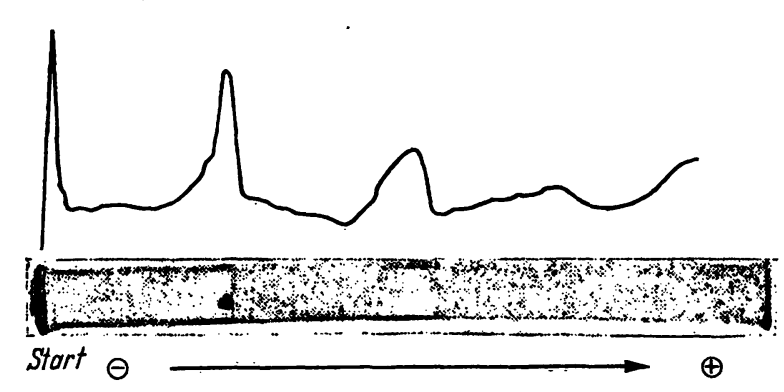

Abb. 8

Diskelektropherogramm und Densitogramm des Serums einer Patientin mit Fernmetastasen eines malignen Tumors (1)

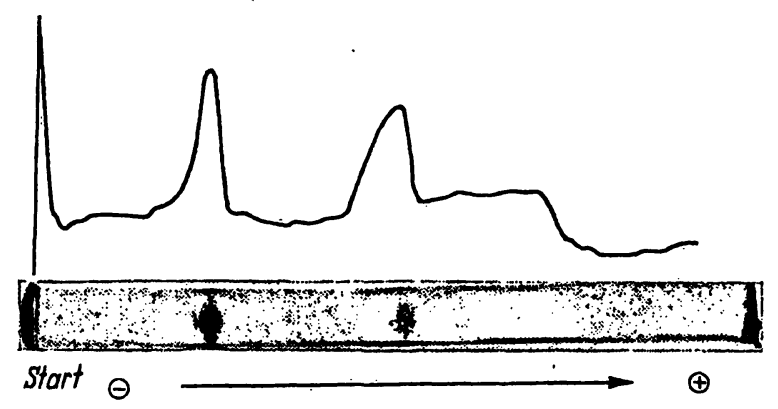

Abb. 9

Diskelektropherogramm und Densitogramm des Serums einer $\mathrm{Pa}$ tientin mit Fernmetastasen eines malignen Tumors (2)

Z. klin. Chem. u. klin. Biochem. / 6. Jahrg. 1968 / Heft 4 


\title{
Literatur
}

1. Davis, B. J. und L. Ornstern, A new high resolution electrophoresis method. Delivered at the Society for the study of blood, New York Academy of Medicine, on the 24th March 1959. 2. RAYMOND, S. und L. S. Weintraub, Science (Washington) 130 , 711 (1959). - 3. Rerspeld, R. A., U. J. Lewis und D. E. WirLIAMS, Nature (London) 195, 281 (1962). - 4. Davis, B. J., Ann. N. Y. Acad. Sci. 121, 404 (1964). - 5. Smithies, O., Adv. Protein Chem. 14, 65 (1959). - 6. OTr, H., Med. Welt, 51, 2697 (1960). 7. Tasweld, H. F. und D. M. Jeffers, Amer. J. Clin. Path. 40, 349 (1963). - 8. Vgl. Sснмidt, E., F. W. Schmidt, H. D. HorN, und U. GerLACH in "Methoden der enzymatischen Analyse“
S. $651-712$, Hrsg. H. U. Bergmeyer, Verlag Chemie GmbH Weinheim/Bergstr. (1962). - 9. Vgl. Hodson, A. W., A. L. Laureen Raine, Clin. chim. Acta (Amsterdam) 7, 255 (1962). 10. Vgl. Estborn, B., diese Z. 2, 53 (1964). - 11. Vgl. Luzzio, A. J., Proc. Soc. Exper. Biol. Med. N. Y. 116, 769 (1964). - 12. Maurer, H.R., Disk-Elektrophorese, W. de Gruyter \& Co. Berlin (1968). - 13. Rosenber G, I. N., J. Clin. Invest. 38, 630 (1959). 14. Kaplan, A. und A. Narahara, J. Laborat. Clin. Med. S. Louis 41, 819 (1953). - 15. Kärcher, K. H. und G. Schulz, Strahlentherapie 131, 395 (1966).

Prof. Dr. K. H. Kärcher 69 Heidelberg, Voßstr. 3

\section{Verfolgung der Zusammensetzung der veresterten Fettsäuren im Kaninchenserum während einer experimentellen alimentären Lipämie}

\author{
Von W. Priz') \\ Aus dem Pbysiologisch-Chemischen und Analytischen Labor (Leiter: Dr. W. Pilz) der Ärztuichen Abteilung \\ (Leiter: Dr. H. Hörlein) der Farbenfabriken Bayer AG, Werk Leverkusen
}

(Eingegangen am 28. Oktober 1967)

Im Anschluß an Untersuchungen über das Arylesterasesystem des Kaninchens wurde die Verteilung der veresterten Fettsäuren bei nüchternen Tieren, bei Tieren mit einer experimentellen alimentären Lipämie und bei solchen, denen Blut 24 Stdn. nach der Lipämie entnommen wurde, untersucht. Mit der präparativen Stärkebreielektrophorese lassen sich veresterte Fettsäuren enthaltende Fraktionen im Bereich des Präalbumins und Albumins, $\operatorname{der} \alpha$-, $\beta$ - und $\gamma$-Globuline isolieren. Im nüchternen Zustand haben alle 4 Fraktionen einen erheblichen Anteil an niederkettigen Fettsäuren, darunter besonders das Präalbumin. Alle 4 Fraktionen sind in der Lage, ihre als Ester gebundenen kurzkettigen Fettsäuren gegen Ester langkettiger Fettsäuren auszutauschen. Im Zustand einer alimentären Lipämie sinkt der Anteil der veresterten niederkettigen Fettsäuren auf ein Minimum ab, um dann später wieder seinen alten Stand annähernd zu erreichen. Es kommen nur jene niedrigen Fettsäuren in veresterter Form vor, die als Phenylester und Tyrosinester Substrate für die Arylesterasen im Kaninchenserum sind. Die Befunde stehen im Einklang mit unserer Theorie, daß der Transport der langkettigen Fettsäuren im Serum über eine von den Arylesterasen katalysierte Umesterung (kurzkettige aromatische Ester + langkettige Fettsäuren = langkettige aromatische Ester + kurzkettige Fettsäuren) erfolgt.

In connection with studies on the aryl esterase system of the rabbit, the distribution of esterified fatty acids was investigated in resting animals and in animals with an experimental alimentary lipaemia, both during and $24 \mathrm{hr}$. after the lipaemia. By preparative starch gel electrophoresis, fractions containing esterified fatty acids were isolated from the areas containing prealbumin and albumin, $\alpha$-, $\beta$ - and $\gamma$-globulins. In the resting state, all 4 fractions contain a high proportion of short chain fatty acids, especially the prealbumin fraction. All 4 fractions will exchange their ester-bound short chain fatty acids with the esters of long chain fatty acids. In alimentary lipaemia, the proportion of esterified short chain fatty acids decreases to a minimum, but the level increases again later and approaches the previous value. The only short chain fatty acids that are found esterified are those which, as phenyl- and tyrosine-esters, act as substrates for the aryl esterases in rabbit serum. These findings agree with our theory that the transport of long chain fatty acids in serum is achieved by a transesterification catalysed by one of the aryl esterases (short chain aromatic ester + long chain fatty acid = long chain aromatic ester + short chain fatty acid).

Bei unseren Studien über die Arylesterasen²) des menschlichen Serums (1) konnten wir feststellen, daß die Arylesterase I durch langkettige Fettsäuren scheinbar gehemmt wird. Wie wir später fanden (2), war diese scheinbare Hemmung durch die Umesterung bedingt. Da bei unseren Methoden zur Bestimmung der Arylesterase (1, 3) das Spaltprodukt $\beta$-Naphthol photometrisch bestimmt wird, war der primäre Eindruck einer Hemmung zu erklären, da kein freies $\beta$-Naphthol bei der Reaktion entsteht. Später fanden wir, $\mathrm{da} \mathrm{B}$ im menschlichen Nabelschnurserum ebenfalls

\footnotetext{
1) Auszugsweise vorgetragen auf der Wintertagung der Deutschen Gesellschaft für Biologische Chemie am 9. 1. 1968 in München. 2) Verwendete Trivialnamen: Arylesterase = Aryl-Ester Hydrolase (EC 3.1.1.2); Clearing Enzyme (12) = endogene Lipoproteidlipase $=$ Glycerin-Ester Hydrolase (EC 3.1.1.3).
}

Arylesterasen enthalten sind, die zum Teil die Fähigkeit zur Umesterung besitzen (4). Schließlich konnten wir nach der Auftrennung eines Vollhomogenates aus Menschen-Leber ein Enzym isolieren, das in der entgegengesetzten Richtung umestert (5). Damit war erwiesen, daß es sich bei den Arylesterasen um Enzyme des Fettstoffwechsels, speziell des Fettsäuretransportes handelt. Das Arylesterasesystem läßt sich wie folgt vereinfacht darstellen (Mensch):

Serum: Tyrosinester niederkettiger Fettsäuren + freie langkettige Fettsäuren $=$ Tyrosinester langkettiger Fettsäuren + freie kurzkettige Fettsäuren.

Leber: Tyrosinester langkettiger Fettsäuren + kurzkettige Fettsäuren = Tyrosinester kurzkettiger Fettșäuren + freie langkettige Fettsäuren. 